\title{
Tratamento endovascular de hemorragia secundária à lesão da artéria esplênica na pancreatite aguda - relato de caso
}

\author{
Endovascular treatment of hemorrhage due to splenic artery injury in \\ acute pancreatitis: case report
}

\author{
Alexandre de Tarso Machado ${ }^{1}$, Ricardo Jayme Procópio ${ }^{1}$, Franco Antônio Cordeiro Neves², \\ Giovanni Menezes Santos ${ }^{3}$, Marcelo Gomes Girundi ${ }^{2}$, Cristina Toledo Afonso ${ }^{4}$, Tulio Pinho Navarro ${ }^{5}$
}

\begin{abstract}
Resumo
A lesão arterial esplênica é uma rara complicação relacionada a situações como trauma, lesão iatrogênica e pancreatite. Hemostasia pode ser feita por identificação do foco do sangramento através de cateterismo seletivo seguido por embolização do ramo sangrante. Relata-se caso clínico de um paciente portador de pancreatite biliar que apresentou, durante procedimento de necrosectomia, hemorragia decorrente da ruptura da artéria esplênica, sendo, então, tratado com sucesso por embolização supersseletiva.
\end{abstract}

Palavras-chave: Pancreatite, hemorragia, embolização.

\section{Introdução}

A ruptura da artéria esplênica como complicação de pancreatite é um evento raro, de causa multifatorial e potencialmente fatal. Angiografia para identificação do local do sangramento seguida por embolização supersseletiva da lesão destaca-se como modalidade terapêutica com alta taxa de sucesso e baixos índices de morbimortalidade.

\section{Descrição do caso}

Homem, 51 anos, sem relato prévio de colelitíase, pancreatite e etilismo, foi admitido com quadro de pancreatite aguda biliar. Recebeu inicialmente tratamento conservador, evoluindo com instabilidade cardiorrespiratória, insuficiência renal, necrose pancreática extensa e aumento da coleção peripancreática. No $16^{\circ}$ dia, foi encaminhado ao bloco cirúrgico e submetido a sequestrectomia de pân-

\begin{abstract}
Splenic artery injury is a rare complication related to trauma, iatrogenic injury, and pancreatitis. Hemostasis can be made by identification of the vascular lesion through selective catheterism followed by embolization of the bleeding vessel. We report a case of a patient with biliary pancreatitis, who presented hemorrhage due to rupture of the splenic artery during a necrosectomy procedure and was successfully treated with selective embolization.
\end{abstract}

Keywords: Pancreatitis, hemorrhage, embolization.

creas com fechamento alternativo de cavidade abdominal com bolsa de Bogotá.

Posteriormente, foram realizadas outras quatro intervenções para revisão de cavidade e desbridamento. Entretanto, durante o último procedimento, ocorreu lesão vascular em região retroperitoneal esquerda com choque hipovolêmico grave. Devido à impossibilidade de hemostasia cirúrgica, optou-se por tamponamento com compressas e indicou-se tratamento endovascular de urgência.

Através de acesso femoral comum direito, foram realizadas aortografia e arteriografias de artéria renal esquerda, artéria gastroduodenal, artéria mesentérica superior e artéria esplênica. Durante estudo angiográfico, evidenciou-se sangramento ativo a partir de lesão complexa (pelo menos em três pontos) no segmento proximal da artéria esplênica (Fi-

\footnotetext{
1. Radiologista intervencionista e cirurgião endovascular. Membro do corpo clínico do Hospital Mater Dei. Belo Horizonte/MG.

2. Cirurgião geral. Membro do corpo clínico do Hospital Mater Dei. Belo Horizonte/MG.

3. Anestesiologista. Membro do corpo clínico do Hospital Mater Dei. Belo Horizonte/MG.

4. Residente de Cirurgia Vascular do Hospital das Clínicas da Universidade Federal de Minas Gerais (UFMG). Belo Horizonte/MG

5. Cirurgião vascular e endovascular. Coordenador do Serviço de Cirurgia Vascular do Hospital das Clínicas da UFMG.

Não foram declarados conflitos de interesse associados à publicação deste artigo.

Artigo submetido em 12.05.09, aceito em 22.10.09.

J Vasc Bras. 2010;9(1):61-65.

Copyright (C 2010 by Sociedade Brasileira de Angiologia e de Cirurgia Vascular
} 
guras 1 e 2). Para minimizar o risco de ruptura ou de destamponamento de potencial coágulo pela manipulação do cateter $5 \mathrm{~F}$, foi realizado cateterismo supersseletivo até o local das lesões com microcateter 2.4F (Progreat ${ }^{\circledR}$, Terumo, Japão) e microfio-guia 0,014" (Terumo, Japão). Para a em-

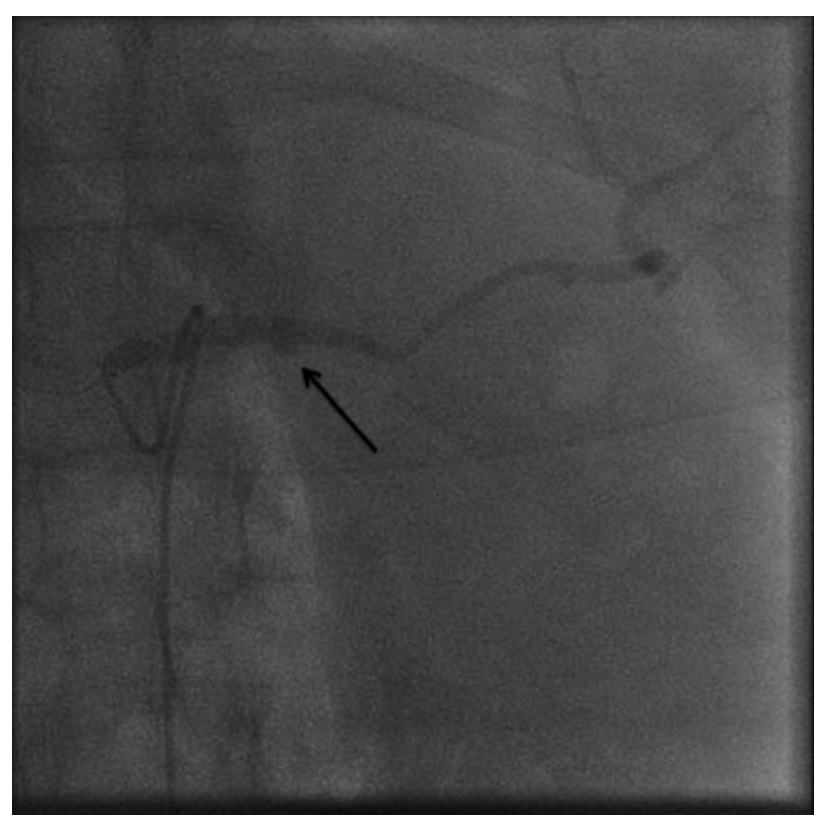

Figura 1 - Arteriografia seletiva do tronco celíaco: artéria esplênica pérvia com pseudoaneurisma identificado no seu segmento proximal, localizando o local do sangramento (seta)

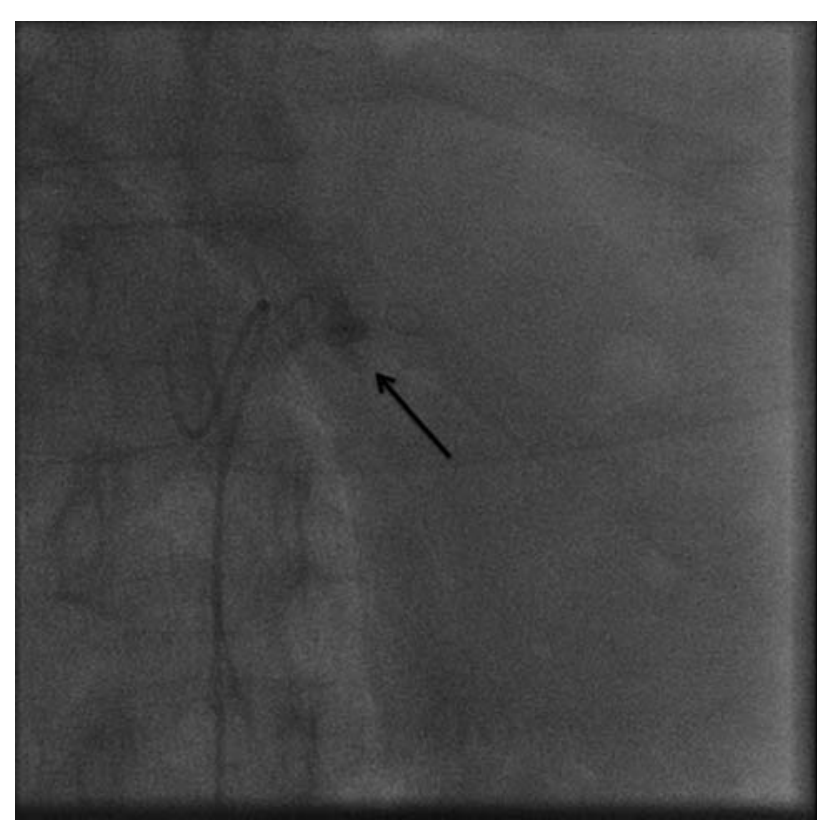

Figura 2 - Arteriografia esplênica: lesão complexa com extravasamento de contraste identificada após cateterismo supersseletivo com microcateter (seta) bolização da artéria esplênica, utilizou-se micromola de 0,018”, medindo 12 × $14 \mathrm{~mm}$ (Nestor ${ }^{\circledR}$, Cook Medical, EUA), que ocupou toda a região lesionada e serviu como arcabouço para o agente tissular n-metil cianoacrilato (Hystoacril $^{\circledR}$, B-Braun, Alemanha) aplicado em seguida na diluição com Lipiodol na concentração de 1:1 (Figuras 3 e 4).

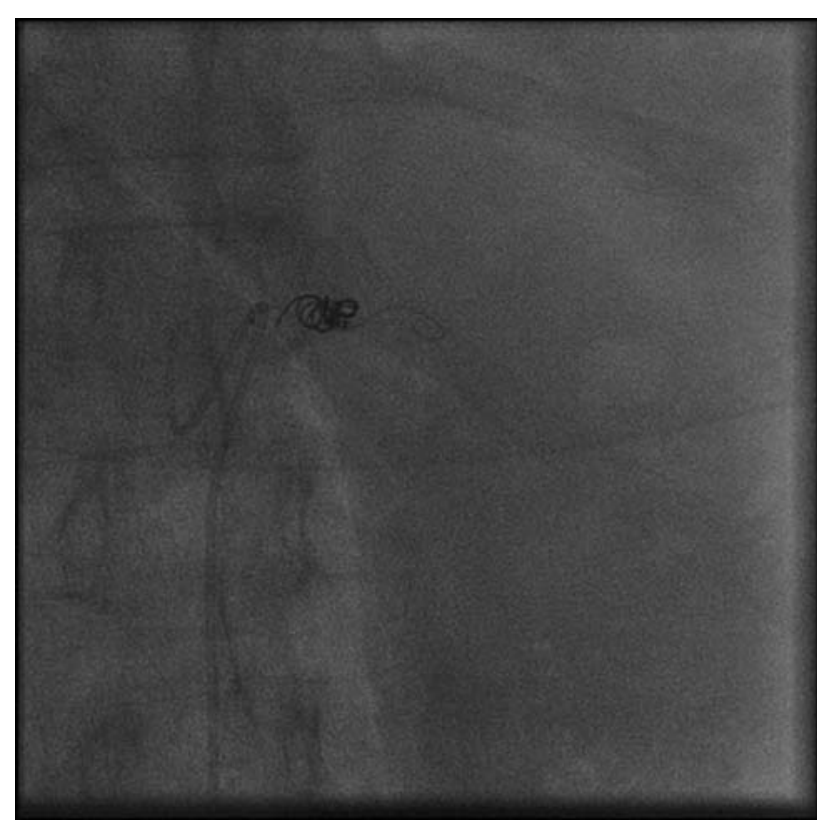

Figura 3 - Liberação da mola ocluindo o segmento proximal da artéria esplênica

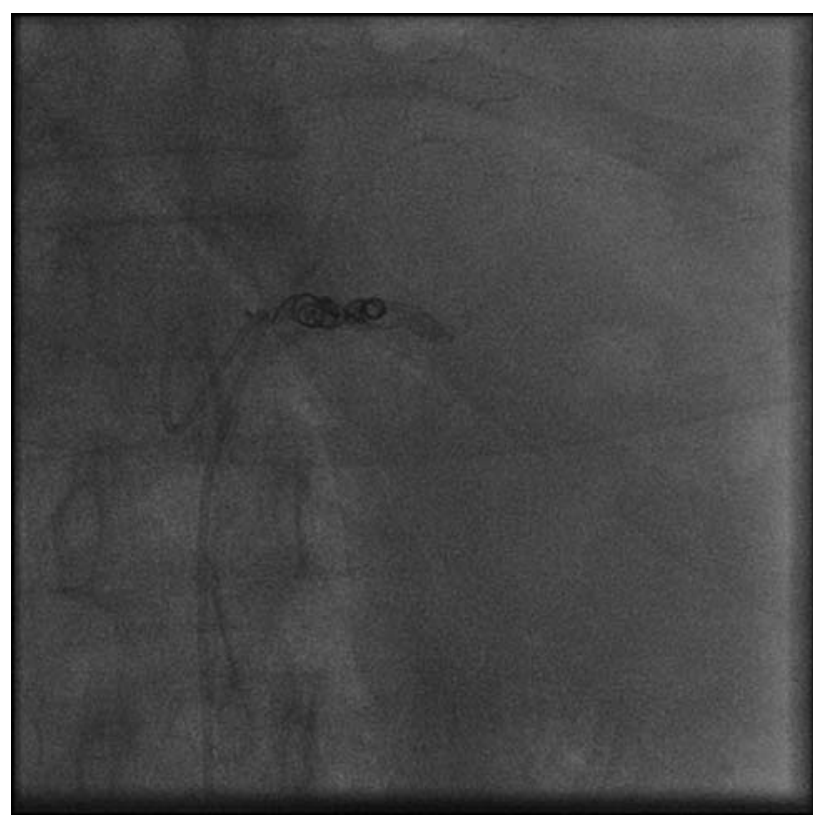

Figura 4 - Aplicação do adesivo tissular (cola) no arcabouço formado pela mola anteriormente posicionada, evitando sua migração distal (material radiopaco) 
O controle angiográfico confirmou oclusão do segmento proximal da artéria esplênica, preservando segmentos distais, sem sinais de extravasamento de contraste (Figura 5).

Durante o período de hospitalização, não ocorreram complicações relacionadas ao procedimento e nem recidiva do sangramento. Entretanto, devido à gravidade da doença de base, o paciente manteve quadro crítico, sendo submetido a mais cinco operações para desbridamento, retirada de compressas e fechamento da laparostomia.

Recebeu alta para enfermaria no $81^{\circ}$ dia de internação hospitalar e alta hospitalar no $124^{\circ}$ dia.

\section{Discussão}

As principais causas de hemorragia decorrente da ruptura da artéria esplênica são: pancreatite, trauma, infecção, úlcera péptica, complicações pós-cirúrgicas e iatrogênicas. No entanto, a maioria dos casos descritos ocorre no contexto de pancreatite ${ }^{1-4}$. Seu diagnóstico e intervenção precoces estão diretamente relacionados com melhores resultados.

A frequência dessas complicações na pancreatite aguda varia entre 1,2 e 14,5\%, sendo menor quando comparada à pancreatite crônica, porém com maiores taxas de mortalidade ( $60 \%$ na aguda contra $22 \%$ na crônica) $)^{6,7}$. As artérias esplênica, gastroduodenal e pancreático-duodenal

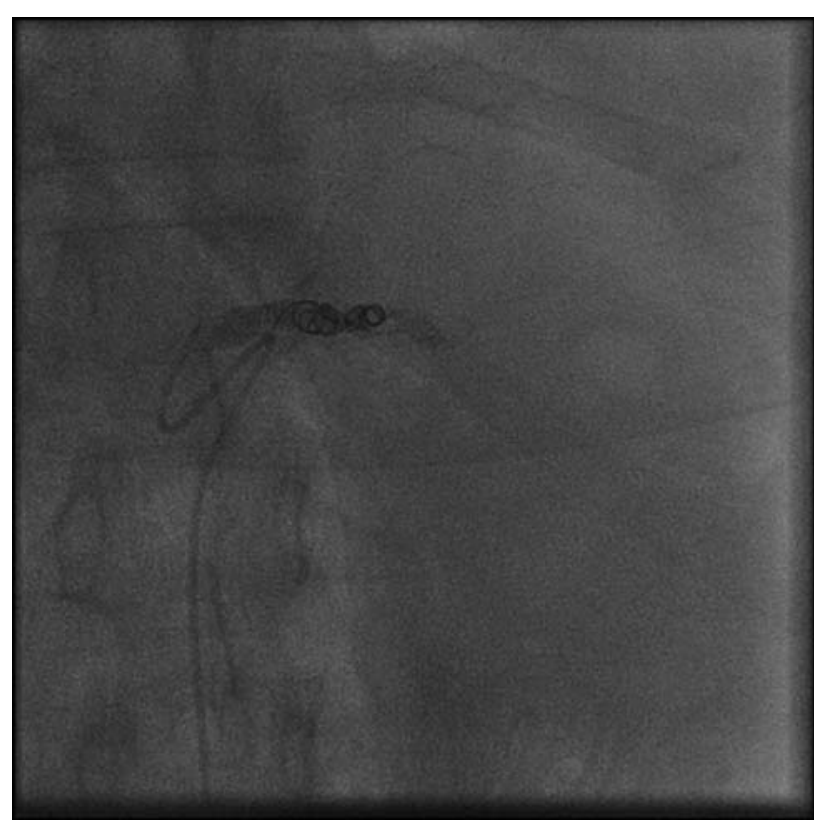

Figura 5 - Arteriografia de controle: oclusão proximal da artéria esplênica sem evidência de extravasamento de contraste são, nessa ordem, os vasos mais comumente envolvidos, estando associados, respectivamente, a taxas de mortalidade de $20,5,27,9$ e $46,1 \% \%^{5,8,9}$.

A patogênese da lesão da artéria esplênica na pancreatite é multifatorial. Um dos seus mecanismos envolve inflamação e consequente necrose pancreática, levando ao extravasamento de enzimas proteolíticas e resultando no enfraquecimento da parede arterial. Outro fator é a técnica operatória traumática, que pode enfraquecer a parede do vaso, expondo-a a futuros danos causados por enzimas ativadas ou corpos estranhos. Também, a utilização prolongada de drenos e compressas intra-abdominais pode agravar o processo. Por fim, outro mecanismo patogênico é a associação com pseudocisto ou abscesso peripancreático, que causa danos vasculares pela combinação de necrose isquêmica, contaminação bacteriana e digestão enzimática pelos seus conteúdos ${ }^{1,2,10}$.

O tratamento pode ser conservador, cirúrgico ou endovascular $^{1,12,13}$. O tratamento endovascular é a abordagem mais segura e eficaz em comparação à cirurgia aberta, com taxa de sucesso de 79 a $100 \%$ e mortalidade entre 12 e $33 \%^{1,2,8,13}$. Na presença de hemorragia, a cirurgia é frequentemente difícil, com anatomia complexa e fonte de sangramento obscura, albergando taxas elevadas de complicações intra e pós-operatórias ${ }^{2,5,8,9}$.

As técnicas endovasculares incluem embolização com Gelfoam, partículas de polivinilalcool (PVA), esferas, molas, balão destacável ou adesivo tissular (cola) $)^{2,11}$. Um risco potencial de se utilizar os agentes particulados (Gelfoam, PVA ou esferas) é que eles podem fluir inadvertidamente para a circulação esplênica distal, causando infarto esplênico ou abscesso ${ }^{14}$.

As molas constituem o agente de escolha para a oclusão dos segmentos proximal e distal à lesão arterial. $\mathrm{Na}$ maioria dos casos, as molas isoladamente são suficientes para produzir o resultado desejado. Contudo, o grande calibre da artéria esplênica pode exigir a utilização de múltiplas molas ou a associação de técnicas com outros agentes embolizantes, como o adesivo tissular (n-metil cianoacrilato), técnica combinada utilizada no caso relatado neste artigo. É importante ressaltar que microcateteres são frequentemente necessários para o posicionamento distal de uma mola ou até mesmo para a cateterização distal de uma artéria tortuosa. O uso de stent revestido, mantendo a luz arterial pérvia, é outra opção de tratamento. Ele permite a 
manutenção da circulação da artéria esplênica; no entanto, a dificuldade técnica inerente à utilização desse dispositivo em um vaso tortuoso e a trombose após o implante do stent são suas maiores limitações ${ }^{1,8,9,11,14}$.

A preservação da artéria esplênica é desejada, porém, há casos de inevitável necessidade de oclusão. Nesses casos, a embolização é feita no local mais próximo da sua origem, pois a circulação sanguínea colateral é geralmente suficiente para suprir o baço, evitando o desenvolvimento de necrose ou abscesso decorrente da isquemia acentuada causada por uma embolização mais distal ${ }^{2,9,14}$.

A ocorrência de complicações após o tratamento endovascular está relacionada com a técnica e materiais utilizados; conhecimento anatômico e da fisiopatologia da doença a ser tratada; e experiência em realizar o procedimento. Quando há complicações, destacam-se principalmente a persistência ou recidiva da hemorragia; síndrome pós-embolização, caracterizada como dor, febre e leucocitose, surgindo logo após o início da embolização e respondendo bem com medidas suportivas; exacerbação da pancreatite; infarto ou abscesso esplênicos; isquemia intestinal; migração do agente embolizante, formação de hematoma ou pseudoaneurisma no local de punção; e insuficiência renal ou alergia induzidos pelo uso do contraste iodado $^{10,11,14}$. A falha em cessar o sangramento pode decorrer do insucesso de cateterizar seletivamente o vaso devido a sua tortuosidade ou vasoespasmo. A recidiva do sangramento após embolização aparentemente bem-sucedida tem sido descrita como devida ao bloqueio incompleto ou recanalização do leito arterial. A reintervenção nessas situações é geralmente bem-sucedida ${ }^{11}$. A persistência de febre e leucocitose pode sugerir abscesso esplênico. $O$ uso adequado de antibióticos associado a fisioterapia ventilatória, evitando que potencial atelectasia na base do pulmão esquerdo se transforme em empiema, são na maioria das vezes eficazes para o controle da infecção. Em algumas situações pode ser necessário realizar a drenagem do abscesso podendo ser feita pelo acesso percutâneo guiado por ultrassom ou tomografia computadorizada. A esplenectomia é indicada nos casos mais graves que não responderam aos tratamentos anteriores ${ }^{6,7,14}$.

\section{Conclusão}

A ruptura da artéria esplênica na pancreatite é uma condição rara e potencialmente fatal. A abordagem endo- vascular permitiu controlar o sangramento decorrente da lesão vascular com bom resultado e baixo risco.

\section{Referências}

1. Hsu JT, Yeh CN, Hung CF et al. Management and outcome of bleeding pseudoaneurysm associated with chronic pancreatitis. BMC Gastroenterol. 2006;6:3.

2. Loffroy R, Guiu B, Cercueil JP et al. Transcatheter arterial embolization of splenic artery aneurysms and pseudoaneurysms: short- and long-term results. Ann Vasc Surg. 2008;22:618-26.

3. Callejas Neto F, Pareja JC, Pilla VF, Leonardi LS. Bleeding complications of chronic pancreatitis: a surgical approach. ABCD Arq Bras Cir Dig. 1994;9:98-101.

4. Leonardi LS. Management of necrotizing hemorrhagic pancreatitis. ABCD Arq Bras Cir. Dig. 1987;2:29-33.

5. Balachandra S, Siriwardena AK. Systematic appraisal of the management of the major vascular complications of pancreatitis. Am J Surg. 2005;190:489-95.

6. Tessier DJ, Stone WM, Fowl RJ et al. Clinical features and management of splenic artery pseudoaneurysm: case series and cumulative review of literature. J Vasc Surg. 2003;38:969-74.

7. Savastano S, Feltrin GP, Antonio T, Miotto D, ChiesuraCorona M, Castellan L. Arterial complications of pancreatitis: diagnostic and therapeutic role of radiology. Pancreas. 1993;8:687-92.

8. Ammori BJ, Madan M, Alexander DJ. Haemorrhagic complications of pancreatitis: presentation, diagnosis and management. Ann R Coll Surg Engl. 1998;80:316-25.

9. Nogara MAS, Cesar AMP, Kanegusuku J, Lopes RW. Hemorragia gastrointestinal alta associada à pancreatite crônica e pseudocisto: relato de um caso / Upper gastrointestinal hemorrhage associated with chronic pancreatitis and pseudocyst: report of a case. Arq Gastroenterol. 1993;30:33-7.

10. Haan JM, Biffl W, Knudson MM et al. Splenic embolization revisited: a multicenter review. J Trauma. 2004;56:542-7.

11. Madoff DC, Denys A, Wallace MJ et al. Splenic arterial interventions: anatomy, indications, technical considerations, and potential complications. Radiographics. 2005;25 Suppl 1:S191-S211.

12. Vujic I, Andersen BL, Stanley JH, Gobien RP. Pancreatic and peripancreatic vessels: embolization for control of bleeding in pancreatitis. Radiology. 1984;150:51-5.

13. Ekeh AP, Izu B, Ryan M, McCarthy MC. The impact of splenic artery embolization on the management of splenic trauma: an 8-year review. Am J Surg. 2009;197:337-41.

14. Piffaretti G, Tozzi M, Lomazzi C, Rivolta N, Riva F, Caronno R, Castelli P. Splenic artery aneurysms: postembolization syndrome and surgical complications. Am J Surg. 2007;193:166-70.

\section{Correspondência:}

Alexandre de Tarso Machado

Av Afonso Pena, 2541

CEP 30130-007 - Belo Horizonte, MG

E-mail:alextm@medicina.ufmg.br 


\section{Contribuições dos autores}

Concepção e desenho do estudo: ATM, FACN, GMS, MGG

Análise e interpretação dos dados: ATM, FACN, GMS, MGG

Coleta de dados: CTA, GMS

Redação do artigo: ATM, RJP, CTA
Revisão crítica do texto: ATM, RJP, TPN

Aprovação final do artigo*: ATM, RJP, FACN, GMS, MGG, CTA, CTN

Análise estatística: N/A

Responsabilidade geral pelo estudo: ATM

Informações sobre financiamento: N/A

* Todos os autores leram e aprovaram a versão final submetida ao J Vasc Bras. 